\title{
Chemical fingerprinting and quantitative analysis of two common Gleditsia sinensis fruits using HPLC-DAD
}

\author{
JIANPING CHEN ${ }^{1}$ \\ ZHONGGUI LI ${ }^{2}$ \\ KEN Y. Z. ZHENG ${ }^{1}$ \\ AVA J. Y. GUO ${ }^{1}$ \\ KEVIN Y. ZHU ${ }^{1}$ \\ WENDY L. ZHANG ${ }^{1}$ \\ JANIS Y. X. ZHAN ${ }^{1}$ \\ TINA T. X. DONG ${ }^{1}$ \\ ZIREN SU ${ }^{3}$ \\ KARL W. K. TSIM ${ }^{1 *}$ \\ ${ }^{1}$ Division of Life Science and Centre for \\ Chinese Medicine, The Hong Kong \\ University of Science and Technology \\ Hong Kong, China \\ 2 School of Health and Pharmacy \\ Guandong Lingnan Institute of \\ Technology, Guangzhou, China \\ 3 School of Chinese Materia Medica \\ Guangzhou University of Chinese \\ Medicine, Guangzhou, China
}

\begin{abstract}
Gleditsiae Fructus Abnormalis and Gleditsiae Sinensis Fructus are obtained from different developmental stages of fruits from Gleditsia sinensis Lam. (Leguminosae). The possible interchangeable usage of the two fruits, however, has long been very controversial. Here, high performance liquid chromatography coupled with diode array detection was developed to explore their chemical fingerprinting profiles. Besides, the amounts of aglycones of saponin compounds, echinocystic acid and oleanolic acid in both fruits were quantified. The results indicated that there was no significant difference in the content of aglycones from the two types of fruits. However, their chromatographic fingerprints showed distinct characteristics. Therefore, the interchangeable application of these fruits has to be taken with a specific precaution.
\end{abstract}

Keywords: Gleditsiae Fructus Abnormalis, Gleditsiae Sinensis Fructus, HPLC-DAD, fingerprinting

Gleditsia sinensis Lam. (Leguminosae) is a saponin-rich plant. Two main types of fruits can be generated from this plant, namely Gleditsiae Sinensis Fructus (GSF), the bigger-sized, normal fruit, and Gleditsiae Fructus Abnormalis (GFA), the abnormal fruit of older or damaged fruits. As they are rich in saponins with antibacterial functions (1-6), both fruits have been widely used in soaps, shampoos, detergents or toothpastes in our daily life. Herbal products containing triterpene saponins of G. sinensis are available in Europe, America, Asia and Africa, and are mainly imported from China where the herb is dominantly cultivated.

\footnotetext{
* Correspondence; e-mail: botsim@ust.hk
} 
J. Chen et al.: Chemical fingerprinting and quantitative analysis of two common Gleditsia sinensis fruits using HPLC-DAD, Acta Pharm. 63 (2013) 505-515.

In the herbal market, the price of GFA is much higher than that of GSF, which is mainly due to the availability of the latter. Thus, herbal traders suggest replacing GFA by GSF for daily application (7). Whether the two fruits could be used in similar applications is still not known. There are many types of triterpenoidal saponins found in GFA or GSF: about 19 saponins have been isolated and identified, which were also reported to possess numerous bioactivities (8-13). The aglycones of these saponins were identified as echinocystic acid and oleanolic acid and were proposed to be active ingredients in numerous medicinal herbs (14-17). At present, the aglycons of saponin compounds are considered to be appropriate chemical compounds for the quality control of herbal medicines, since they are convenient in terms of method development and marker availability (18-20). Besides, HPLC fingerprint has been considered to be a comprehensive approach for assessing the quality of herbal medicines (21). Today, this technique is widely used for quality control and species differentiation of herbs $(22,23)$. We therefore employed the HPLC fingerprint and quantitative analysis approaches to reveal the variation between the two fruits.

\section{EXPERIMENTAL}

\section{Chemicals and materials}

Twelve herb samples were collected from different geographical regions of China. Ten batches of GFA and two batches of GSF were collected from the same plants. Plant materials were authenticated according to their morphological characteristics. Voucher specimens were deposited in the Centre for Chinese Medicine at Hong Kong University of Science \& Technology, Hong Kong, China.

The chemical standards of echinocystic acid and oleanolic acid were purchased from the ChromaDex (ChromaDex, USA) and the National Institute for the Control of Pharmaceutical \& Biological Products (NICPBP, China). Their purity was determined to be over $98 \%$ by normalization of peak areas, as revealed by HPLC-DAD.

Gleditsioside A was isolated from the roots of GFA, and its structure was established by comparison of its spectral data (mass spectrum, ${ }^{1} \mathrm{H}$ NMR and ${ }^{13} \mathrm{C}$ NMR) with the literature data $(8,12)$. In general, the purity of a chemical marker should be over $98 \%$, which could be used for quantification. As for gleditsioside A, the main saponin in the fruits, used as a marker for HPLC fingerprint, was purified in-house. This chemical was rather unstable during storage, and the purity was around $95 \%$. Thus, this chemical could be used as a reference standard for qualitative HPLC fingerprint only.

HPLC-grade acetonitrile was purchased from Merck (Germany) and ultra-pure water was prepared using a Milli-Q purification system (Millipore S.A.S., France). Other reagents used were of analytical grade.

\section{Preparation of sample solutions}

Stock solutions of echinocystic acid, oleanolic acid and gleditsioside A were prepared in concentrations of 1000, 800 and $200 \mathrm{mg} \mathrm{L}^{-1}$, respectively, by accurately weighing 
J. Chen et al.: Chemical fingerprinting and quantitative analysis of two common Gleditsia sinensis fruits using HPLC-DAD, Acta Pharm. 63 (2013) 505-515.

each compound into a volumetric flask and dissolving it in methanol. Stock solutions were stored at $-20{ }^{\circ} \mathrm{C}$.

GFA was pulverized and dried to constant mass and then passed through a $0.25-\mathrm{mm}$ sieve before use. For fingerprint analysis, $0.1 \mathrm{~g}$ of sample was accurately weighed and placed into a 15-mL Falcon tube and extracted with $10 \mathrm{~mL} 25 \%$ methanol in an ultrasonic bath for $30 \mathrm{~min}$. The solution was filtered through a $0.45-\mu \mathrm{m}$ Millipore filter before injection of $10 \mu \mathrm{L}$ into the HPLC.

For quantitative analysis, $0.5 \mathrm{~g}$ of herbal powder was accurately weighed and placed into a $250-\mathrm{mL}$ round-bottomed flask, reflux was extracted with $25 \mathrm{~mL}$ of methanol for $30 \mathrm{~min}$, the solution was transferred into a 50-mL centrifuge tube and centrifuged at $4,000 \times \mathrm{g}$ for $5 \mathrm{~min}$. Ten $\mathrm{mL}$ of the supernatant was pipetted into a $250-\mathrm{mL}$ round-bottomed flask and concentrated to dryness under vacuum using a rotary evaporator. The residue was dissolved in $10 \mathrm{~mL}$ of diluted hydrochloric acid/ethanol solution and reflux was extracted for $30 \mathrm{~min}$. After cooling, the solution was transferred into a $15-\mathrm{mL}$ centrifuge tube and centrifuged at $4,000 \times g$ for $5 \mathrm{~min}$. The supernatant was discarded and the residue was transferred to a $25-\mathrm{mL}$ volumetric flask and made up to the mark with methanol. The solution was filtered through a $0.45-\mu \mathrm{m}$ Millipore filter before HPLC analysis.

\section{Apparatus and chromatographic conditions}

The HPLC system consisted of an Agilent 1200 binary pump (Agilent, Germany), an auto sampler and a DAD detector at $210 \mathrm{~nm}$ for all analyses. Chromatographic separation was carried out on an Alltima $C_{18}$ column $(4.6 \times 250 \mathrm{~mm}, 5 \mu \mathrm{m})$. For fingerprint analysis, the mobile phase consisted of acetonitrile (A) and water (B) using the following gradient program: $0-60 \mathrm{~min}, 25.0-40.0 \%$ A. A pre-equilibration period of $10 \mathrm{~min}$ was used between individual runs. For quantitative analysis, the mobile phase consisted of acetonitrile and water using the following gradient program: 0-10 $\mathrm{min}, 65.0 \% \mathrm{~A}, 10-20$ min, $65.0-85.0 \% \mathrm{~A}, 20-30 \mathrm{~min}, 85 \% \mathrm{~A}$. A pre-equilibration period of $10 \mathrm{~min}$ was used between individual runs. The column temperature was $25{ }^{\circ} \mathrm{C}$ with a flow rate of $1.0 \mathrm{~mL}$ $\mathrm{min}^{-1}$ at $210 \mathrm{~nm}$ and the injection volume of $10 \mu \mathrm{L}$.

For the MS analysis, an Agilent QQQ-MS/MS (6410B, Germany) equipped with an ESI (electrospray ionization) ion source was operated in positive ion mode. The drying gas, nitrogen temperature and flow were $325^{\circ} \mathrm{C}$ and $10 \mathrm{~L} \mathrm{~min}^{-1}$, respectively, nebulizer pressure was $241 \mathrm{kPa}$, capillary voltage $4.0 \mathrm{kV}$; delta electro multiplier voltage $400 \mathrm{~V}$. Agilent Mass Hunter workstation software version B.01.00 was used for data acquisition and processing.

\section{Method validation $(18,19,24)$}

Linearity. - Linearity was established by injection of $10 \mu \mathrm{L}$ of mixed standard solution at six different concentrations. Calibration curve was plotted subsequently based on linear regression analysis of the integrated peak vs. concentration $\left(\mu \mathrm{g} \mathrm{mL} \mathrm{m}^{-1}\right)$. Each calibration curve included six data points. 
J. Chen et al.: Chemical fingerprinting and quantitative analysis of two common Gleditsia sinensis fruits using HPLC-DAD, Acta Pharm. 63 (2013) 505-515.

Limit of detection and limit of quantification. - The limits of detection (LOD) and quantification $(L O Q)$ were determined at a signal-to-noise ratio $(\mathrm{S} / \mathrm{N})$ of 3 and 10 , respectively.

Precision. - The precision of the method was validated by determining of intra- and inter-day variability. The intra- and inter-day precision was determined by analyzing six replicates of the standard solution of the two analytes during a single day and six replicates of the sample examined on 3 successive days, respectively. The relative standard deviation (RSD) was taken as a measure of intermediate precision.

Recovery. - A recovery test was used to evaluate the accuracy of method. An exact amount of each standard was added to accurately weighed $0.5 \mathrm{~g}$ of GFA (sample C), which was then extracted and analysed as described. For comparison, a blank sample (not spiked with standard compounds) was prepared and analyzed.

HPLC fingerprinting. - In HPLC fingerprinting, a well resolved peak corresponding to an available chemical reference substance can be used as a marker peak for the calculation of RRTs (relative retention times) of other peaks in the same chromatogram. The RRT of a characteristic peak is calculated retention time of a chosen marker peak. For positive identification, the sample must give all characteristic peaks with the RRTs falling within the acceptable range.

\section{RESULTS AND DISCUSSION}

\section{Extraction procedure and chromatographic conditions}

The contents of echinocystic acid and oleanolic acid (Fig. 1a) were determined in a hydrochloric acid hydrolysed herbal extract. Fig. 1b shows a typical HPLC profile of mixed standards and an extract of GFA or GSF at $210 \mathrm{~nm}$. In order to obtain a quantitative extraction of analytes from GFA or GSF, the variables involved in the extraction procedure were optimized. Different extraction solvents (methanol, $70 \%$ methanol, absolute ethanol and $70 \%$ ethanol) and different concentrations of hydrochloric acid/50 \% ethanol $\left(0.5,1.0\right.$ and $\left.2.0 \mathrm{~mol} \mathrm{~L}^{-1} \mathrm{HCl}\right)$ were tested. Extraction by methanol and hydrolysis by $2 \mathrm{~mol} \mathrm{~L}-1 \mathrm{HCl} / 50 \%$ ethanol were found to be optimal (Fig. 2). Different ratios of acetonitrile/water and methanol/water were investigated for the optimal mobile phase, and the quantitative analysis was achieved by gradient elution using a binary mixture of acetonitrile and water.

\section{Method validation}

Linearity. - Linearity was observed in the concentration range $1.0-800 \mu \mathrm{g} \mathrm{mL}^{-1}$ for echinocystic acid and 1.9-1000 $\mu \mathrm{g} \mathrm{mL}^{-1}$ for oleanolic acid. Calibration curves depict excellent correlations for echinocystic acid $\left(R^{2}=0.9998\right)$ and oleanolic acid $\left(R^{2}=0.9990\right)$.

$L O D$ and LOQ. - The LODs for two analytes were 0.05 and $0.06 \mu \mathrm{g} \mathrm{mL}^{-1}$. Their LOQs were 1.00 and $1.50 \mu \mathrm{g} \mathrm{mL}-1$, respectively. 
a)

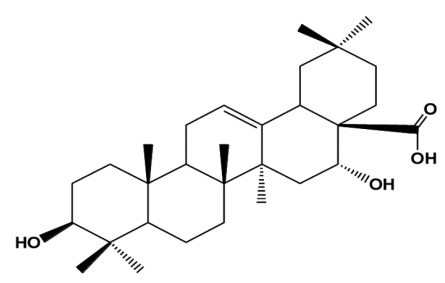

Echinocystic acid

b)

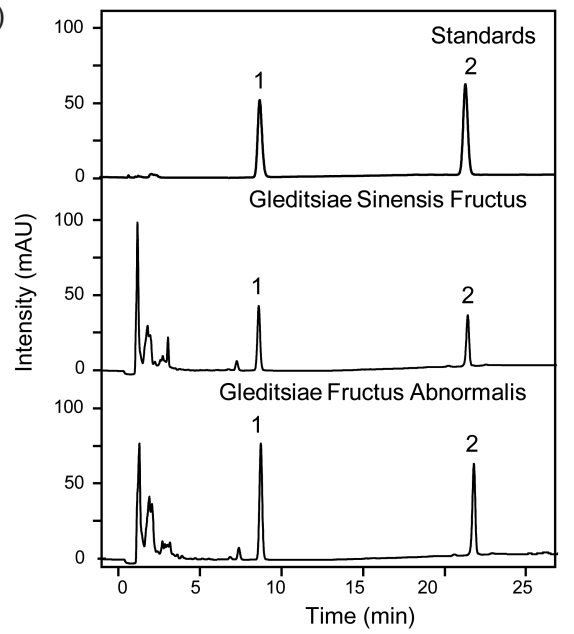

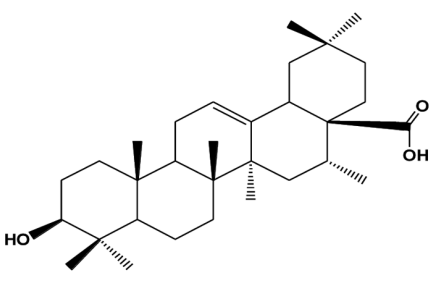

Oleanolic acid

Fig. 1. a) Chemical structures of echinocystic acid and oleanolic acid analyzed in Gleditsiae Fructus Abnormalis and Gleditsiae Sinensis Fructus. b) Representative HPLC chromatograms of mixed standards and extract of Gleditsiae Fructus Abnormalis and Gleditsiae Sinensis Fructus. 1 echinocystic acid, 2 - oleanolic acid.

Precision. - Precise values evaluated on model samples were as follows. The intra-day RSD values for two analytes were 0.7 and $0.4 \%(n=6)$, and inter-day RSD values were 3.8 and $3.4 \%$, respectively $(n=6)$ (Table I).

Recovery. - The average recoveries $(n=6)$ for echnocystic acid and oleanolic acid were $95.8 \pm 2.3 \%$ and $93.2 \pm 2.2 \%$, respectively (Table I).

Results indicated that the developed HPLC-DAD method was precise, accurate and sensitive enough for simultaneous quantitative evaluation of echinocystic acid and oleanolic acid in GFA.

\section{Determination of echinocystic acid and oleanolic acid}

The validated HPLC-DAD method was applied to quantify two chemical markers, echinocystic acid and oleanolic acid, in 12 batches of samples. The results of quantitative analyses are summarized in Table II. We found that GSF (K and L) contained both echinocystic acid and oleanolic acid. The average contents of echinocystic acid for GFA and GSF were $19.76 \pm 4.52$ and $19.70 \pm 1.67 \mathrm{~g} \mathrm{~kg}^{-1}$, respectively, while those of oleanolic acid were $21.25 \pm 1.95 \mathrm{~g} \mathrm{~kg}^{-1}$ and $15.38 \pm 0.86 \mathrm{~g} \mathrm{~kg}^{-1}$, respectively. As described, GSF (K and 
Fig. 2. Comparison of different hydrolytic concentrations of hydrochloric acid during extraction. Values are expressed in $\mathrm{g} \mathrm{kg}^{-1}$ of dried single herb. Mean $\pm \mathrm{SD}, n=3$. Significant difference: ${ }^{*} p<0.05$, ${ }^{* *} p<0.01$.

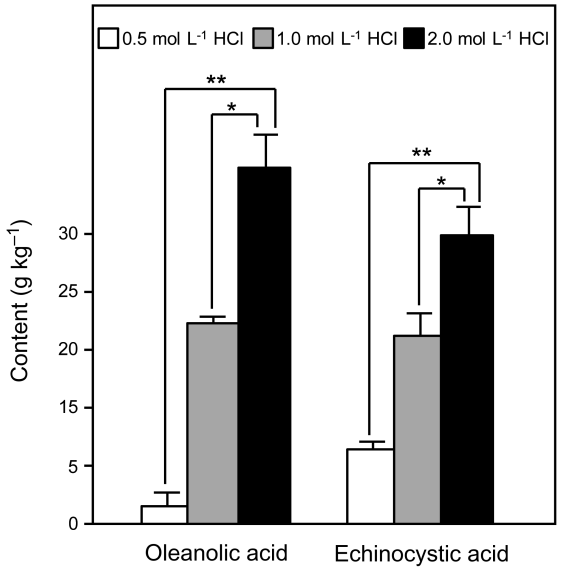

L) and GFA (E and J) were collected from the same plants, yet the amount of aglycons hydrolyzed from saponins was different. Specifically, GFA (E) with echinocystic acid to oleanolic acid ratio was 1.1, while GSF (K) with two markers ratio was 1.3. Likewise, the ratios of two markers were 1.0 and 1.3, respectively. These results suggested a different ratio of saponins contained in two different types of fruits: GSF contained a higher ratio of echinocystic acid-type saponins to oleanolic acid-type ones than GFA. Similarly, GFA contained a higher ratio of oleanolic acid-type saponins to echinocystic acid-type ones than GSF. We speculated that these different ratios of both analytes in two fruits, this might be due to the different developmental stages of the fruit. A research for saponins by Xia et al. (24) reported that the contents in GFA were higher than that in GSF, and their ratios were $2.4: 1.8: 0.9: 1.0$ and $2.3: 2.3: 1.0: 1.0$ for GFA and GSF, respectively. Indeed, a research by $\mathrm{Li}$ and $\mathrm{Hu}(25)$ reported that the amount of oleanolic acid decreased during the development of fruits. However, further study should be conducted to investigate how these two analytes changed in the fruits.

Table I. Precision, repeatability and recovery of two analytes in model samples

\begin{tabular}{|c|c|c|c|c|c|c|}
\hline \multirow{3}{*}{ Analyte } & \multicolumn{4}{|c|}{ Precision } & \multicolumn{2}{|c|}{ Recovery $(n=6)$} \\
\hline & \multicolumn{2}{|c|}{ Intra-day $(n=6)$} & \multicolumn{2}{|c|}{ Inter-day $(n=6)^{\mathrm{a}}$} & \multirow{2}{*}{$\begin{array}{l}\text { Mean } \\
(\%)\end{array}$} & \multirow{2}{*}{$\begin{array}{l}\text { RSD } \\
(\%)\end{array}$} \\
\hline & $\begin{array}{c}\text { Mean } \\
\left(\mu \mathrm{g} \mathrm{mL} \mathrm{mL}^{-1}\right)\end{array}$ & $\begin{array}{c}\text { RSD } \\
(\%)\end{array}$ & $\begin{array}{c}\text { Mean } \\
\left(\mu \mathrm{g} \mathrm{mL}^{-1}\right)\end{array}$ & $\begin{array}{l}\text { RSD } \\
(\%)\end{array}$ & & \\
\hline Echinocystic acid & 30.10 & 0.2 & 29.32 & 1.1 & 95.85 & 2.3 \\
\hline Oleanolic acid & 27.40 & 0.1 & 26.83 & 0.9 & 93.23 & 2.2 \\
\hline
\end{tabular}

a The inter-day analysis refers to six replicates of the sample examined on three consecutive days. 
J. Chen et al: Chemical fingerprinting and quantitative analysis of two common Gleditsia sinensis fruits using HPLC-DAD, Acta Pharm. 63 (2013) 505-515.

Table II. Content of echinocystic acid and oleanolic acid in Gleditsiae Fructus Abnormalis and Gleditsiae Sinensis Fructus

\begin{tabular}{ccccc}
\hline \multirow{2}{*}{ Item } & Sample & $\begin{array}{c}\text { Production/ } \\
\text { collection area }\end{array}$ & \multicolumn{2}{c}{ Content $\left(\mathrm{g} \mathrm{kg}^{-1}\right)^{\mathrm{a}}$} \\
\cline { 4 - 5 } & & & Echinocystic acid & Oleanolic acid \\
\hline A & $\begin{array}{c}\text { Gleditsiae Fruc- } \\
\text { tus Abnormalis }\end{array}$ & Shandong (HK Market) & $17.81 \pm 2.31$ & $22.78 \pm 2.37$ \\
$\mathrm{~B}$ & Ditto & Shandong (HK Market) & $20.99 \pm 2.86$ & $22.67 \pm 0.61$ \\
$\mathrm{C}$ & Ditto & Shandong (HK Market) & $27.95 \pm 1.73$ & $19.22 \pm 2.68$ \\
$\mathrm{D}$ & Ditto & Shandong (HK Market) & $12.91 \pm 3.42$ & $19.42 \pm 1.91$ \\
$\mathrm{E}$ & Ditto & Guangzhou, Guangdong & $24.18 \pm 1.82$ & $21.51 \pm 0.48$ \\
$\mathrm{~F}$ & Ditto & Yuncheng, Shanxi & $21.05 \pm 2.60$ & $24.08 \pm 2.83$ \\
$\mathrm{G}$ & Ditto & Anguo, Hebei & $15.46 \pm 0.46$ & $18.22 \pm 1.33$ \\
$\mathrm{H}$ & Ditto & Taian, Shandong & $16.44 \pm 3.21$ & $20.24 \pm 2.08$ \\
$\mathrm{I}$ & Ditto & Shangqiu, Henan & $17.88 \pm 0.11$ & $21.12 \pm 1.43$ \\
$\mathrm{~J}$ & Ditto & Lintong, Shanxi & $22.93 \pm 3.13$ & $23.24 \pm 3.03$ \\
$\mathrm{~K}$ & Gleditsiae Sinensis & Guangzhou, & $20.88 \pm 2.23$ & $15.99 \pm 0.81$ \\
$\mathrm{~L}$ & Fructus & Guangdong & $18.52 \pm 0.92$ & $14.77 \pm 1.69$ \\
\hline
\end{tabular}

a Values are expressed in $\mathrm{g} \mathrm{kg}^{-1}$ of dried fruit mass.

Mean $\pm \mathrm{SD}, n=3$.

Although the literature demonstrates that echinocystic acid, one of the aglycones, was employed to quantitatively control the quality of GFA $(26,27)$, previous methods, especially for sample preparation, were time-consuming, i.e. around 20 hours. Besides, hazardous solvents such as chloroform were used in the extraction. In comparison with these methods, our current method is simpler and more practical to control the quality of the fruits. For instance, the preparation procedure could be done within 3 hours. Moreover, less hazardous solvent was employed in this study.

\section{HPLC fingerprint analysis}

In order to clarify the chemical properties of the two fruits, chromatograms of different samples were generated. The process of standardization included the selection of »characteristic peaks « in chromatograms and normalization of retention times of all characteristic peaks. Gleditsioside A, one of the major saponins found in both fruits, was used as the reference standard in the fingerprint analysis. Due to similar physicochemical properties of saponins, in this paper we identified the reference peak in samples by comparing the HPLC retention time and MS data in positive mode with the reference standard. In the MS spectra of samples, the molecular ion of $[\mathrm{M}+\mathrm{Na}]^{+}$at $\mathrm{m} / \mathrm{z} 1643.0$ was deduced from gleditsioside A, which further confirmed the identity of gleditsioside A (Fig. 3a). As shown in Fig. 3b, the chromatograms of both fruits from different regions contained 5 characteristic peaks. Among these peaks, gleditsioside A (peak 5), which 


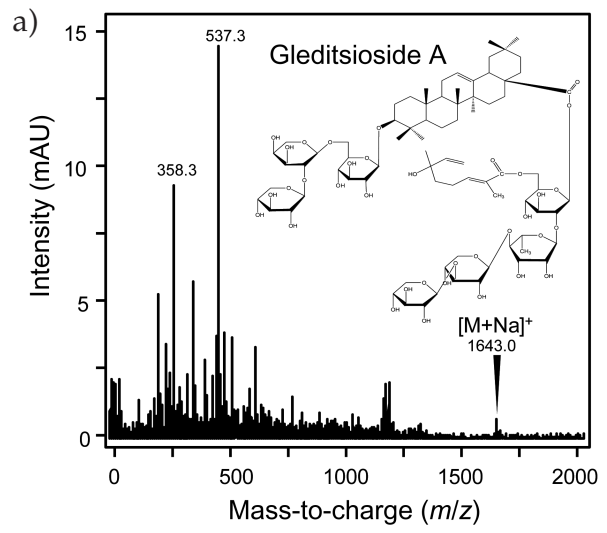

c)
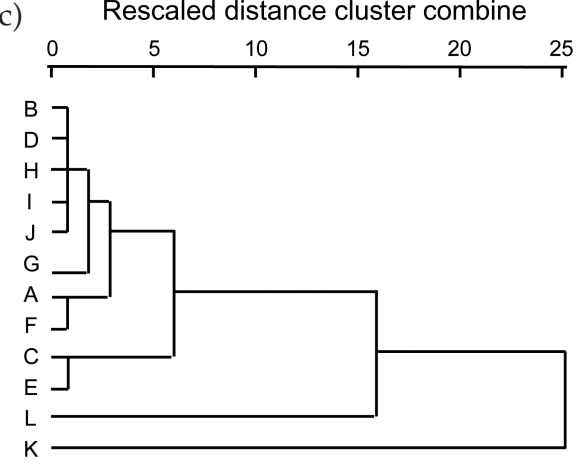

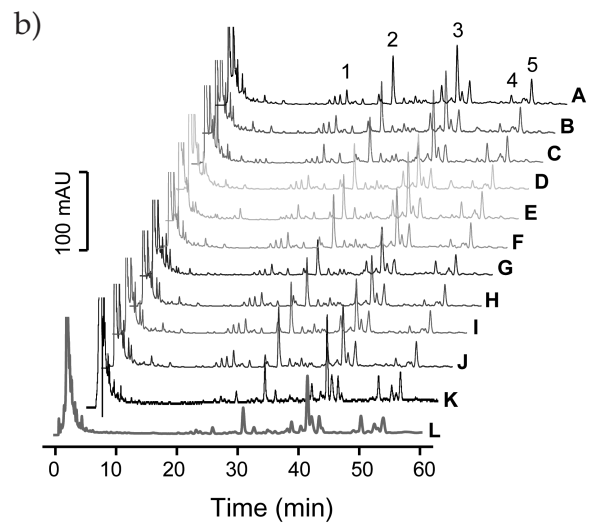

Fig. 3. HPLC fingerprint of Gleditsiae Fructus Abnormalis and Gleditsiae Sinensis Fructus. a) Chemical structure of gleditsioside A extracted from Gleditsiae Fructus Abnormalis and Gleditsiae Sinensis Fructus. b) HPLC fingerprints of twelve samples (letters A to L stand for different samples shown in Table II). Peaks 1-5 are defined as characteristic peaks and peak 5 (gleditsioside A) serves as the reference peak. c) Hierarchical clustering analysis for twelve samples. The loading plot was performed with the original peak areas of five common peaks as input data.

eluted at a retention time of $54.7 \mathrm{~min}$, was chosen as the marker peak. The relative retention time $(R R T)$ of the 5 characteristic peaks with reference to peak 5 (gleditsioside A) were: for peak $1(0.42 \pm 0.11), 2(0.57 \pm 0.02), 3(0.77 \pm 0.06), 4(0.94 \pm 0.02)$. The RSD values of RRTs of the 5 peaks in 12 sample batches were less than $3.0 \%$, which confirmed the high stability and reproducibility of the fingerprints.

In the hierarchical clustering analysis, method called average linkage between groups was applied, and Pearson correlation was selected as measurement. The result is shown in Fig. 3c. The tested populations of fruits were divided into two main clusters: samples A-J (GFA) as cluster one and samples K and L (GSF) as cluster two. This clustering agreed well with the results of two distinct types of fruits. The results here suggested that the difference between two fruits might be the result from the different ratios of saponins between two developmental stages. This is in agreement with the study reporting that saponin contents could be changed due to the developmental stages of herbs (25).

Both fruits were recognized as the same herbal medicine in Compendium of Materia Medica by Li Shizhen in the Ming dynasty (A.D. 1578) of China. However, GFA was de- 
J. Chen et al.: Chemical fingerprinting and quantitative analysis of two common Gleditsia sinensis fruits using HPLC-DAD, Acta Pharm. 63 (2013) 505-515.

scribed as a drug of higher quality than GSF. Nowadays, they are recorded as different medicinal drugs in Chinese pharmacopoeia (18). In line with this, the study by Xiao (28) has suggested that GFA and GSF cannot be substitutes for each other because of the difference of their growth habitats as well as their chemical compositions.

As regards to the content of total saponins, no significant difference was found between GFA and GSF $(26,29)$. Total amounts of four saponins, including gleditsioside A, gleditsioside $\mathrm{B}$, gleditsioside D and gleditsioside I were 52.7 and $52.4 \mathrm{mg} \mathrm{g}^{-1}$ for GFA and GSF, respectively (24). However, some findings revealed that there were differences between two fruits when comparing the amount of echinocystic acid and metals, i.e., $\mathrm{Cu}$, $\mathrm{Zn}, \mathrm{Fe}, \mathrm{Mn}, \mathrm{Mg}$, $\mathrm{K}$ and $\mathrm{Ca}$, as well as their antibacterial effect $(26,30)$. Here, we found that the chemical fingerprints of both fruits are rather different, and therefore the interchangeable usage of GFA and GSF as a medicine should be undertaken with precaution, unless full chemical composition has been revealed.

\section{CONCLUSIONS}

The established HPLC-DAD method was found to be accurate and precise enough to be adopted for routine quality control of GFA and GSF. GSF was found to be very similar to GFA in the content of echinocystic acid and oleanolic acid. On the other hand, HPLC fingerprinting revealed a different ratio of echinocystic acid to oleanolic acid type saponins. Further chemical and pharmaceutical study should be conducted to reveal whether GFA might be replaced by GSF in clinical applications.

Acknowledgments. - This research was supported by the Hong Kong Research Grants Council Theme-based Research Scheme (T13-607/12R) and GRF (661110, 662911, 663012), and Foundation of The Awareness of Nature (TAON12SC01) to KWKT. JPC received a scholarship from the Hong Kong Chiu Chow Chamber of Commerce. This work is part of a study of some common Chinese Material Medica in Hong Kong undertaken by the Department of Health, Hong Kong SAR Government, China.

\section{REFERENCES}

1. G. Francis, Z. Kerem, H. P. Makkar and K. Becker, The biological action of saponins in animal systems: A review, Br. J. Nutr. 88 (2002) 587-605; DOI: 10.1079/BJN2002725.

2. S. G. Sparg, M. E Light and J. van Staden, Biological activities and distribution of plant saponins, J. Ethnopharmacol. 94 (2004) 219-243; DOI: 10.1016/j.jep.2004.05.016.

3. J. D. Zhang, Y. B. Cao, Z. Xu, H. H. Sun, M. M. An, L. Yan, H. S Chen, P. H. Gao, Y. Wang, X. M. Jia and Y. Y. Jiang, In vitro and in vivo antifungal activities of the eight steroid saponins from Tribulus terrestris L. with potent activity against fluconazole-resistant fungal pathogens, Biol. Pharm. Bull. 28 (2005) 2211-2215; DOI: 10.1248/bpb.28.2211.

4. B. Yu, Y. Zhang and P. Tang, Carbohydrate chemistry in the total synthesis of saponins, Eur. J. Org. Chem. 31 (2007) 5145-5161; DOI: 10.1002/ejoc.200700452.

5. Y. F. Chen, C. H. Yang, M. S. Chang, Y. P. Ciou and Y. C. Huang, Foam properties and detergent abilities of the saponins from Camellia oleifera, Int. J. Mol. Sci. 11 (2010) 4417-4425; DOI: 10.3390/ ijms11114417. 
J. Chen et al.: Chemical fingerprinting and quantitative analysis of two common Gleditsia sinensis fruits using HPLC-DAD, Acta Pharm. 63 (2013) 505-515.

6. X. Crebassa, H. Caruel, F. Mercier and G. R. M. G. San Martin, Use of Saponines from Gleditsia Against Mollusc Pests, E.P. Pat. 2,533,646, 29 Dec 2012.

7. X. P. Yang, A comparative study of chemical components of Fructus Gleditsiae Sinensis and Fructus Gleditsiae Abnormalis, J. Huaiyin Teachers Coll. (Nat. Sci. Ed.), 2004, 143-146.

8. Z. Zhang, K. Koike, Z. Jia, T. Nikaido, D. Guo and J. Zheng, Gleditsiosides N-Q, new triterpenoid saponins from Gleditsia sinensis, J. Nat. Prod. 62 (1999) 877-881; DOI: 10.1021/np990060m.

9. L. M. Chow, J. C. Tang, I. T. Teo, C. H. Chui, F. Y. Lau, T. W. Leung, G. Cheng, R. S. Wong, I. L. Wong, K. M. Tsang, W. Q. Tan, Y. Z. Zhao, K. B. Lai, W. H. Lam, D. A. Guo and A. S. Chan, Antiproliferative activity of the extract of Gleditsia sinensis fruit on human solid tumour cell lines, Chemotherapy 48 (2002) 303-308; DOI: 10.1159/000069713.

10. Y. Dai, Y. P. Chan, L. M. Chu and P. P. Bu, Antiallergic and anti-inflammatory properties of the ethanolic extract from Gleditsia sinensis, Biol. Pharm. Bull. 25 (2002) 1179-1182; DOI: 10.1248/ bpb.25.1179.

11. L. J. Fu, Y. Dai, Z. T. Wang and M. Zhang, Inhibition of experimental allergic rhinitis by the n-butanol fraction from the anomalous fruits of Gleditsia sinensis, Biol. Pharm. Bull. 26 (2003) 974-977; DOI: 10.1248/bpb.26.974.

12. L. F. Hou, Y. Dai, Y. F. Xia and Z. N. Gong, Alleviation of picryl chloride-induced delayed type hypersensitivity reaction by saponin fraction of Gleditsia sinensis, Biol. Pharm. Bull. 29 (2006) 1056-1059; DOI: 10.1248/bpb.29.1056.

13. Z. Z. Gao, Y. F. Xia, X. J. Yao, Y. Dai and Q. Wang, A new triterpenoid saponin from Gleditisia sinensis and structure-activity relationships of inhibitory effects on lipopolysaccharide-induced nitric oxide production, Nat. Prod. Res. 22 (2008) 320-332; DOI: 10.1080/14786410701766422.

14. X. Tong, S. Lin, M. Fujii and D. X. Hou, Molecular mechanisms of echinocystic acid-induced apoptosis in HepG2 cells, Biochem. Biophys. Res. Commun. 321 (2004) 539-546; DOI: 10.1016/j. bbrc.2004.07.004.

15. B. Plohmann, G. Bader, K. Hiller and G. Franz, Immunomodulatory and antitumoral effects of triterpenoid saponins, Pharmazie 52 (1997) 953-957.

16. J. Liu, Pharmacology of oleanolic acid and ursolic acid, J. Ethnopharmacol. 49 (1995) 57-68; DOI: 10.1016/0378-8741(95)90032-2.

17. C. Gauthier, J. Legault, K. Girard-Lalancette, V. Mshvildadze and A. Pichette, Hemolytic activity, cytotoxicity and membrane cell permeabilization of semi-synthetic and natural lupane- and oleanane-type saponins, Bioorg. Med. Chem. 17 (2009) 2002-2008; DOI: 10.1016/j.bmc.2009.01.022.

18. Pharmacopoeia of the People's Republic of China, The State Pharmacopoeia Commission of P. R. of China, Beijing 2010.

19. Hong Kong Chinese Materia Medica Standards, Department of Health, Government of the Hong Kong Special Administrative Region, Hong Kong 2010, pp. 203-204.

20. Z. Q. Yin, R. F. Zhang, J. Zhang, M. Kong and Y. Dai, Quality evaluation of Gleditsiae Fructus Abnormalis, J. China Pharm. Univ. 42 (2011) 428-430.

21. Y. Z. Liang, P. S. Xie and K. Chan, Chromatographic fingerprinting and metabolomics for quality control of TCM, Comb. Chem. High T. Scr. 13 (2010) 943-953; DOI: 10.2174/138620710793360 310 .

22. W. Lu, F. Yang and S. Wang, Development of an HPLC fingerprint for quality control and species differentiation of Uncaria rhynchophylla (Miq.) ex Havil, Acta Chromatogr. 24 (2012) 643-651; DOI: 10.1556/AChrom.24.2012.4.10.

23. Q. F. Zhang, H. Y. Cheung and L. B. Zeng, Development of HPLC fingerprint for species differentiation and quality assessment of Rhizoma Smilacis Glabrae, J. Nat. Med. 67 (2013) 207-211; DOI: $10.1007 / \mathrm{s} 11418-012-0648-9$. 
J. Chen et al: Chemical fingerprinting and quantitative analysis of two common Gleditsia sinensis fruits using HPLC-DAD, Acta Pharm. 63 (2013) 505-515.

24. Y. F. Xia, Z. Z. Gao, Y. Dai and Q. Wang, Analysis of bioactive saponins in Fructus Gleditsiae Abnormalis and Fructus Gleditsiae Sinensis by LC-ELSD, Chromatographia 70 (2009) 1361-1366; DOI: $10.1365 /$ s10337-009-1312-8.

25. J. T. Li and Z. H. Hu, Accumulation and dynamic trends of triterpenoid saponin in vegetative organs of Achyranthus bidentata, J. Integr. Plant Biol. 51 (2009) 122-129; DOI: 10.1111/j.1744-7909. 2008.00764.x.

26. N. Li, W. F. Bao and G. Song, The quantitative determination of echinocystic acid in Gleditsia sinensis L. by HPLC, J. Shenyang Pharm. Univ. 18 (2010) 198-200.

27. J. H. Qu, Z. H. Li and P. Li, Determination of echinocystic acid in Fructus Gleditsiae Abnormalis from different regions by HPLC, China Pharmacist 13 (2010) 385-386.

28. Y. J. Xiao, Gleditsiae Sinensis Fructus should not be confused with Gleditsiae Fructus Abnormalis, Capital Med. 16 (2009) 49.

29. Y. Y. Jia and G. F. Zhang, Comparison of total saponins between Gleditsia Sinensis Fructus and Gleditsiae Fructus Abnormalis, J. Chin. Med. Mat. 4 (1987) 19-21.

30. Y. Y. Jia, S. Z. Wang and G. F. Zhang, Preliminary comparative analysis of the fruit of Gleditsia sinensis Lam. and G. officinalis Hemsl., China J. Chin. Mat. Med. 12 (1987) 35-37. 